\title{
Anisotropic Self-Assembly and Gelation in Aqueous Methylcellulose-Theory and Modeling
}

\author{
Valeriy V. Ginzburg, ${ }^{1}$ Robert L. Sammler, ${ }^{1}$ Wenjun Huang, ${ }^{2}$ Ronald G. Larson ${ }^{2}$ \\ ${ }^{1}$ The Dow Chemical Company, Core R\&D, Materials Science, Midland, Michigan 48674 \\ ${ }^{2}$ Department of Chemical Engineering, University of Michigan, Ann Arbor, Michigan 48109 \\ Correspondence to: V. Ginzburg (E-mail: vvginzburg@dow.com)
}

Received 16 February 2016; accepted 28 March 2016; published online 19 April 2016

DOI: $10.1002 /$ polb.24065

ABSTRACT: Recent experimental studies demonstrated that the aqueous methylcellulose (MC) polymer chains in water can form nanoscale fibrils (diameter $\sim 14 \mathrm{~nm}$, persistence length $\sim 60 \mathrm{~nm}$ ), and those fibrils can organize into networks at higher temperatures and/or concentrations, forming the commonly observed gel. Here we propose that the fibrils are onedimensional self-assemblies of stacked, fused polymer rings that are formed at elevated temperatures due to the changing nature of the MC-water hydrogen bonding. This mechanism is analogous to the coil-helix transition in polypeptides, although it is not clear whether the MC fibrils possess chirality. We perform coarse-grained molecular simulations of $\mathrm{MC}$ chain struc- ture at temperatures both above and below the hypothesized coil-to-ring transition, with CG forcefield tuned by atomistic molecular dynamics simulations, and observe the expected conformational change. We then develop a statistical mechanical theory to predict the fibril self-assembly, gelation and rheology as function of temperature and concentration. The findings are in reasonable agreement with experimental data and could be generalized to other carbohydrate polymers. (c) 2016 Wiley Periodicals, Inc. J. Polym. Sci., Part B: Polym. Phys. 2016, 54, 1624-1636

KEYWORDS: gelation; methylcellulose; simulation; theory
INTRODUCTION Cellulosic ether (CE) materials are widely used in a variety of market segments including pharmaceutical, food, construction, personal care, industrial specialties, and many others. ${ }^{1,2}$ They are based on the most abundant and renewable raw material in the world: cellulose. A key CE material of interest is methylcellulose (MC), Figure 1(a), because of its unique properties which include being nontoxic, soluble in cold water, and gelling in hot water. Due to this range of properties, MC has found widespread use in many applications. Over the years, a number of studies have been devoted to the phase behavior of aqueous MC. ${ }^{3-15}$ Very recently, studies by Bodvik et al., ${ }^{16}$ and independently by Kobayashi et al. ${ }^{17-22}$ provided unexpected evidence of anisotropic polymer selfassembly into high-aspect-ratio fibrils of MC. In particular, Bates et al. used a combination of experimental techniques (SANS, cryo-TEM, optical, and rheology) to find the following features for a set of water-soluble MC materials (polymer concentration in water ranged from 0.014 to 12 wt \%) prepared by the same commercial heterogeneous process with similar degrees of methylether substitution $(1.8 \mathrm{~mol} \mathrm{MeO} / \mathrm{mol}$ anhydro glucose unit (AGU)). The materials transformed from a cold clear fluid to a hot turbid hydrogel when warmed, and returned to a cold clear fluid when again cooled. The tempera- ture of the gelation transition during warming was insensitive of the MC molecular weight $\left(M_{\mathrm{w}}=59-530 \mathrm{~kg} / \mathrm{mol}\right)$ at any given MC concentration, and decreased as MC concentration rose. The transition temperatures were reported at very slow warming rates of $2{ }^{\circ} \mathrm{C} / \mathrm{h}$ to approximate equilibrium conditions desirable for phase diagrams. The presence of fibrils was confirmed using cryo-TEM and small-angle neutron scattering. Moreover, key features of the fibril structure include a diameter of $14 \pm 2 \mathrm{~nm}$, high aspect ratio, Kuhn length of $50 \pm 5 \mathrm{~nm}$, and water level of $60 \pm 5 \%$, which were reported to be insensitive to MC molecular weight or concentration or solution temperature. The fibrils are reminiscent of wormlike surfactant micelles, ${ }^{23}$ one-dimensional assemblies of "hairy" nanoparticles $^{24}$ and attractive colloidal spheres, ${ }^{25}$ or liquid crystal defect-mediated amphiphile assemblies. ${ }^{26}$ At high temperatures, fibrils assemble into hydrogels with fairly high elastic shear storage modulus, $G$ '. Depending on the temperature and concentration (but not polymer molecular weight), $G^{\prime}$ is in the $10^{3}$ to $10^{5} \mathrm{~Pa}$ range, far above what would be expected for a polymer entanglement network at a similar concentration. ${ }^{18,19}$

To explain this behavior, we hypothesize that methylcellulose chains in water undergo a conformational transition similar

Additional Supporting Information may be found in the online version of this article.

(c) 2016 Wiley Periodicals, Inc. 


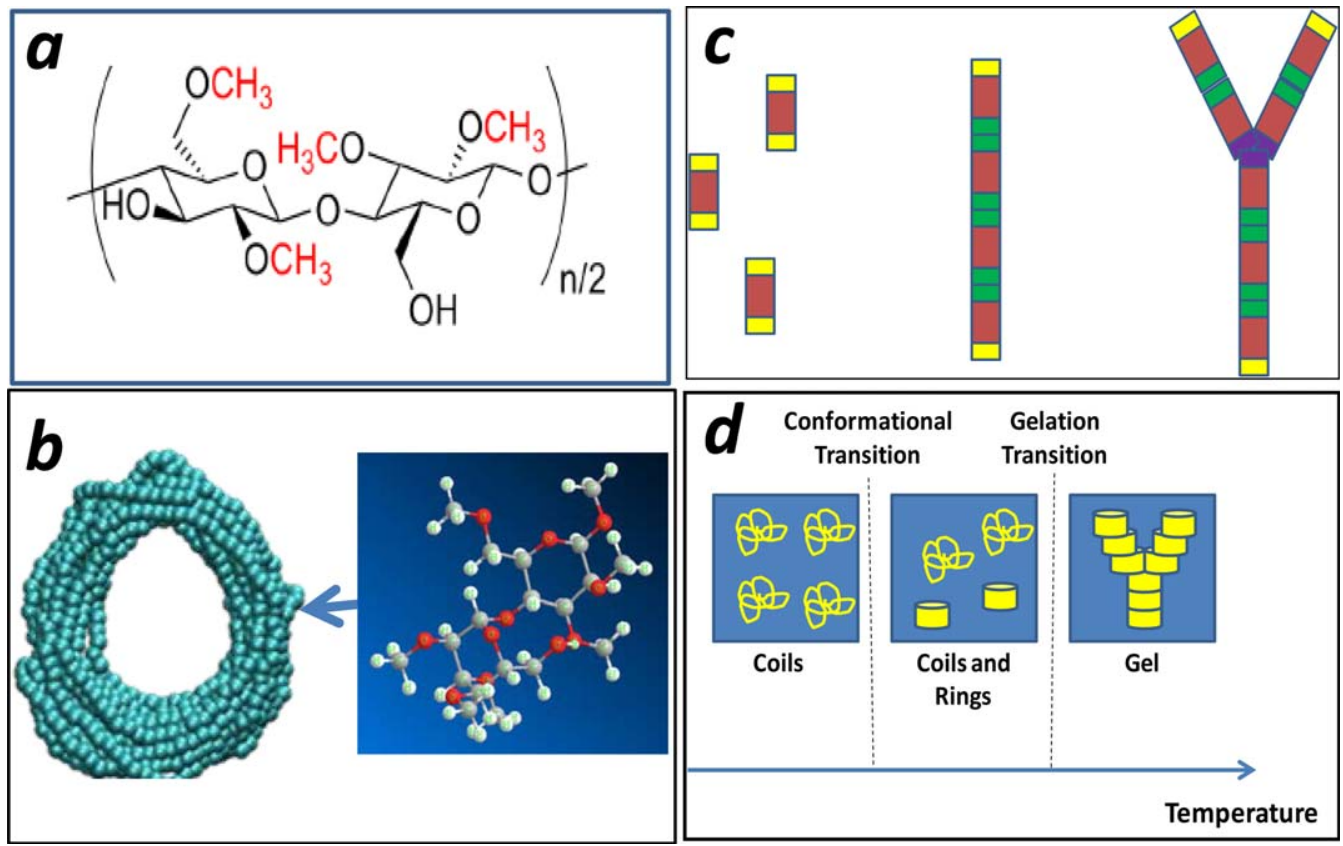

FIGURE 1 (a) Chemical structure of methylcellulose (MC) with degree of substitution DS of $2.0 \mathrm{~mol}$ MeO/mol AGU. (b) Proposed schematics of the ring configuration (higher temperatures). (c) Individual rings (left), tubes (middle), and Y-junctions (right). (d) Proposed phase diagram of aqueous MC as function of temperature. Conformational transition from coils to rings occurs at lower temperatures, followed by the self-assembly of the rings into hollow fibrils and formation of network of fibrils (gelation). [Color figure can be viewed in the online issue, which is available at wileyonlinelibrary.com.]

to a coil-helix transition in polypeptides. ${ }^{27-36}$ The coil-helix transition has been the subject of many studies over the past half-century. Zimm and Bragg, ${ }^{27}$ and Lifson and Roig ${ }^{28}$ proposed simple theories of this transition based on an Ising-like two state model. Baur and Nosanow ${ }^{29,30}$ discussed the origins of both normal (helix at low temperatures, coil at high temperatures) and inverted (coil at low temperatures, helix at high temperatures) coil-helix transitions and calculated the enthalpy and entropy change for this transition as function of the effective hydrogen bonding parameters. Formation of helix structures-as well as intermediate metastable structures such as "racquets" - were also modeled in a number of coarsegrained simulations ${ }^{35,37-39}$ of semi-flexible polymers with attractive interactions. Very recently, Huang et al. ${ }^{40}$ used coarse-grained molecular dynamics (CG-MD) to show that at elevated temperatures, aqueous $\mathrm{MC}$ chains adopt a ring-like conformation [Fig. 1(b)]. This transition is caused by temperature-induced changes in the relative strength of cellulose-water and cellulose-cellulose hydrogen bonds. ${ }^{41}$ Lott et al. and Guillot et al. have considered the possibility that MC fibrils observed in their experiments could be helices, but pointed out the lack of light depolarization that should be seen for helical structures. ${ }^{19,42}$ This objection could be overcome, though, if the fibril is chiral only on a local scale with handedness varying randomly over larger scales, or if each molecule winds up in a ring structure with no observable chirality, as indeed is suggested by simulations.

The above discussion was mainly about the configuration of a single chain. If it is granted that a single chain can form a ring-like structure, as indicated by simulations (and reinforced by simulations presented here), then the next step is to consider association of rings into fibrils i.e., physical "polymerization." Once a ring structure is formed from one or a few chains, it is proposed here that the rings will reduce their free energy the most by stacking on top of other rings to assemble end-to-end into long one-dimensional hollow fibrils [Fig. 1(c)]. Moreover, sometimes nonlinear assemblies of ends are expected, enabling the formation of threedimensional (3D) fibril networks. An example of a nonlinear trifunctional fibril network junction (Y-branch) is also illustrated in Figure 1(c). A theoretical framework describing the anisotropic self-assembly of fibrils and networks was developed by Zilman et al.; ${ }^{43-46}$ using their approach, one can describe or predict the average aspect ratio of a selfassembled fibril and the density of "junctions" as function of temperature and concentration. The overall phase behavior, then, looks as shown in Figure 1(d): a solution of expanded coils at low temperatures; coexistence of coils and rings at intermediate temperatures; and ultimately a 3D network of high-aspect-ratio tubes at high temperatures.

The last step in the modeling is describing the rheology. As already discussed by Arvidson et al., ${ }^{18-21}$ the elasticity of the MC fibrillar network at high temperature should be similar to that of the actin network or any other network of semiflexible fibers. The elasticity of semi-flexible fibrillar network is often described by the model of MacKintosh et al., ${ }^{4-49}$ resulting in a strong dependence of the shear storage modulus, $G^{\prime}$, on the polymer concentration, $c$, as $G^{\prime} \sim c^{2.5}$ This 


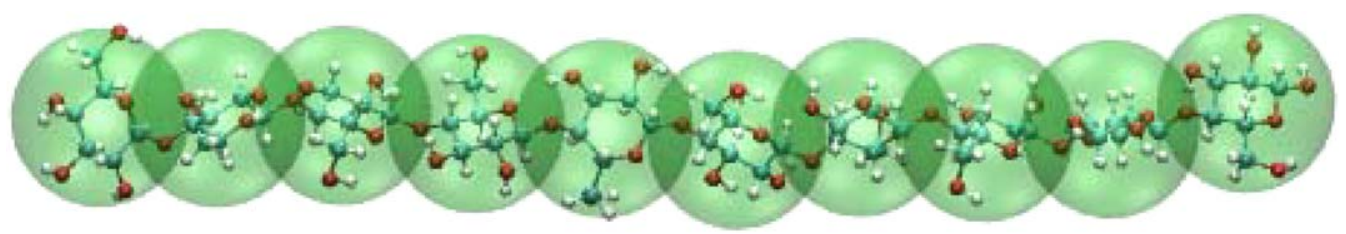

FIGURE 2 Schematic depiction of the methylcellulose coarse-grained model. Each methylcellulose repeat unit (DS ranging from 0 to 3 ) is represented by one bead centered at the repeat unit center-of-mass (COM). The beads are connected via harmonic springs. [Color figure can be viewed in the online issue, which is available at wileyonlinelibrary.com.]

result is indeed consistent with experiments for the case of gelatin networks, as demonstrated by Joly-Duhamel et al. ${ }^{50}$ We use the same modeling approach to compute the dependence of $G^{\prime}(T)$ for various concentrations of aqueous MC, while estimating the crosslink density as function of temperature from thermodynamic theory. The proposed approach, to the best of our knowledge, is the first model able to summarize the self-assembly process of aqueous MC chains into fibrils. It is capable of describing key experimental results and trends and potentially can be generalized to other types of cellulose ethers and other carbohydrate polymers.

The paper is structured as follows. First, we use coarse-grained molecular dynamics (CG-MD) and demonstrate that at elevated temperatures, single-chain $\mathrm{MC}$ rings can assemble into onedimensional "proto-tubes." Next, we present our statistical mechanical model describing the self-assembly of the tubes into networks ("gelation") and the gel rheology. The results are then compared with the experimental data of Arvidson et al., ${ }^{18}$ and a good semi-quantitative agreement is found.

\section{THEORY}

\section{Coarse-Grained Molecular Dynamics (CD-MD)}

The CG-MD model used in this work is described in detail by Huang et al. in a separate publication. ${ }^{40}$ Here we are outlining a few details of the model. The simulations were performed with Brownian dynamics (BD) simulation technique in an implicit solvent environment with the LAMMPS simulation package ${ }^{51}$ (ver. Feb 2014) using the NVE ensemble. The CG simulations were set up with reduced LJ (Lennard-Jones) units, where all quantities were dimensionless, and were related to three fundamental units, mass $(m)$, distance $(\sigma)$, and energy $(\varepsilon)$, which were all set to unity. To convert to dimensional real units, we chose the unit mass $(m)$ to be $188 \mathrm{amu}(\mathrm{g} / \mathrm{mol})$, unit distance $(\sigma)$ to be $0.515 \mathrm{~nm}$, and unit energy $(\varepsilon)$ to be $1 k_{\mathrm{b}} T$ at $298 \mathrm{~K}(2.48 \mathrm{~kJ} / \mathrm{mol})$. The simulations were coupled with a Langevin thermostat with temperature maintained at unity in reduced temperature units $(298 \mathrm{~K})$. We ran simulations at several "effective temperatures" in this study, where the effect of the temperature was captured by varying the strength of the intermolecular interaction. The unit time $(\tau)$ was estimated to be $0.028 \mathrm{~ns}$ by comparing the polymer self-diffusivity calculated from CG simulations using mean-square displacement calculation and that estimated from experiments using the Stokes law for an equivalent sphere of radius comparable to radius of gyration of the polymer. A typical CG simulation in this study uses a time step of $0.0005 \tau$ (14 fs) and runs for $2 \times 10^{8}$ time steps $(2.8 \mu \mathrm{s})$. The data presented in this work are averaged over at least $5 \times 10^{7}$ time steps $(>700 \mathrm{~ns})$.

The CG methylcellulose chains were modeled with beads and stiff springs, with each bead representing a methylcellulose repeat unit (Fig. 2). Both bonded and nonbonded interactions were included in our CG polymer model, and the parameters of these were obtained from by fitting the intramolecular repeat unit center-of-mass (COM) radial distribution functions (RDFs) generated from the single-chain atomistic simulations of homogenous methylcellulose oligomers with degree of polymerization $\mathrm{DP}=10$. Homopolymers of all eight repeat units of water-soluble methylcellulose were simulated. Since intramolecular structure does not vary much with substitution pattern, these eight intramolecular repeat unit COM RDFs were averaged to produce a single RDF, and the CG bonded parameters were chosen so that the peak positions and height were matched between the CG intramolecular bead-bead RDF and atomistic intramolecular repeat unit COM RDF [Supporting Information, Fig. S1(a)]. On the other hand, intermolecular interactions are expected to be sensitive to the substitution pattern. Hence, for each of the eight substitution types, the nonbonded parameters were obtained by separately fitting the intermolecular repeat unit COM-repeat unit COM RDF generated from an atomistic simulation containing multiple identical homogenous methylcellulose chains (DP $=10-30$, concentration $10 \mathrm{wt} \%)$ using a modified Iterative Boltzmann Inversion technique. ${ }^{40,52,53}$ The parameter fitting was performed for each of the eight atomistic systems at two temperatures (25 and $50{ }^{\circ} \mathrm{C}$ ), and therefore 16 sets of nonbonded interaction pairs were obtained.

The complete CG interaction potential was expressed by the following equation

$$
U_{\mathrm{CG}}=U_{\mathrm{bond}}+U_{\text {angle }}+U_{\text {dihedral }}+U_{\text {nonbonded }}
$$

The bonded interaction included harmonic bond, angle, and dihedral interactions, which were applied to any two, three, and four consecutive beads on a chain respectively,

$$
\begin{gathered}
U_{\text {bond }}=1 / 2 K_{\mathrm{b}}\left(l-l_{0}\right)^{2} \\
U_{\text {angle }}=1 / 2 K_{\theta}\left(\theta-\theta_{0}\right)^{2} \\
U_{\text {dihedral }}=K_{\varphi}[1+\operatorname{dcos}(n \varphi)]
\end{gathered}
$$

Here $l_{0}$ and $\theta_{0}$ are the equilibrium bond length and angle, and $K_{\mathrm{b}}$ and $K_{\theta}$ the corresponding bond and angle force 
constants. In the dihedral expression, $d$ and $n$ are the phase constants, and $K_{\varphi}$ is the dihedral force constant.

The nonbonded interactions were parameterized using a truncated and shifted Lennard-Jones 9-6 potential,

$$
U_{\text {nonbonded }}(r)=\left\{\begin{array}{l}
4 \varepsilon_{i i}\left[\left(\frac{\sigma_{i i}}{r}\right)^{9}-\left(\frac{\sigma_{i i}}{r}\right)^{6}-\left(\frac{\sigma_{i i}}{r_{c}}\right)^{9}+\left(\frac{\sigma_{i i}}{r_{c}}\right)^{6}\right] r<r_{c} \\
0 r \geq r_{c}
\end{array}\right.
$$

Three parameters $\left(\varepsilon_{i i}, \sigma_{i i}\right.$, and $\left.r_{c_{\mathrm{ii}}}\right)$ were obtained for each of the eight methylcellulose repeat unit substitution types to reflect the different solvation behavior of each type. The parameters for each substitution type were first obtained for homogenous short chains ( $\mathrm{DP} \leq 30$ ) by matching the position and height of the first peak (which occurs roughly at $r=0.6 \mathrm{~nm}$ ) of an atomistic intermolecular repeat unit COM RDF with that of the intermolecular CG RDF [Supporting Information, Fig. S1(b)]. ${ }^{40}$ We then kept the $\sigma_{i i}$, and $r_{c_{\mathrm{ii}}}$ values constant for different chain lengths of each repeat unit substitution type, but allowed the energy parameter $\epsilon_{i i}$ to depend on chain length. We used a series of short-chain results to perform a power law extrapolation $\left(\epsilon_{i i}=A(\mathrm{DP})^{-B}\right)$ of the $\varepsilon_{i i}$ value up to $\mathrm{DP}=1000$. In our previous work, ${ }^{40}$ it was shown that the prediction of the methylcellulose persistence length and chain collapse transition using these extrapolated values are in a good agreement with experimental results. In that study, we used randomly substituted $\mathrm{DP}=1000$ polymer chains; therefore, the $\epsilon_{i i}$ values used here are the ones obtained by setting DP $=1000$. Finally, we used a geometric mixing rule (eq 1f) to calculate the $\varepsilon_{i j}$ and $\sigma_{i j}$ values between methylcellulose repeat units of differing methyl substitution.

$$
\begin{aligned}
\sigma_{i j} & =\sqrt{\sigma_{i i} * \sigma_{j j}} \\
\varepsilon_{i j} & =\sqrt{\varepsilon_{i i} * \varepsilon_{j j}}
\end{aligned}
$$

We parameterized three sets of intermolecular interaction parameters for each of the eight methylcellulose substitution types. The three sets correspond to three different temperatures, namely $25{ }^{\circ} \mathrm{C}$ (low temperature), $50{ }^{\circ} \mathrm{C}$ (high temperature), and an intermediate temperature (approximately $40{ }^{\circ} \mathrm{C}$ ), with $50{ }^{\circ} \mathrm{C}$ being close to the typical gelation temperature for dilute (1-2 wt \%) methylcellulose solutions. ${ }^{18}$ Note here that while the parameter sets for 25 and $50{ }^{\circ} \mathrm{C}$ were obtained based on the parameterization procedure described above, the parameter set for the intermediate temperature was obtained by lowering the $\varepsilon_{i i}$ values for all eight repeat unit substitution types at $50{ }^{\circ} \mathrm{C}$ by $20 \%$, which, based on a linear interpolation between the high-temperature and low-temperature parameter values, corresponds to a temperature of around $40{ }^{\circ} \mathrm{C}$. We have summarized all CG-MD parameters in Supporting Information Table S1 (for bonded interactions) and Table S2 (for nonbonded interactions). We note that the intermolecular interaction parameters used in the implicit-solvent model are derived from intermolecular monomer RDFs generated from explicit-solvent atomistic simulations, and therefore the effect of the solvent is embedded implicitly in these RDFs. As discussed in previous work, ${ }^{40}$ some explicit entropic contributions from the solvent are neglected by removing the water from the model, which might be crucial for properly explaining the fibril structure formation in dense solution. However, simulating long chains (DP $>50$ ) using explicit-solvent atomistic model is computationally impossible at current stage, and a typical coarsegrained explicit-solvent model that lumps a few water molecules into a water bead is too coarse for reproducing local water structures around the polymer chain. Therefore, we will leave it to future work to provide a coarse-grained model that can model the effect of solvent more accurately.

Our earlier CG-MD study ${ }^{40}$ of a single heterogeneous MC chain DP $=600$ showed that upon heating, there was a conformational transition from a coil (at $25{ }^{\circ} \mathrm{C}$ ) to a ring (at 50 ${ }^{\circ} \mathrm{C}$ ). The repeat unit substitution type distribution-representing "ideal" water-soluble MC chains commonly associated with commercial heterogeneous processes - is given in Supporting Information, Table S3. In the next section, we will demonstrate that the ring structure remains stable in systems with multiple MC chains (at least for relatively low polymer concentrations), and that the rings assemble into proto-tubes which then can grow into hollow fibrils. We now turn our attention to statistical mechanics of fibril selfassembly and gelation.

\section{Statistical Mechanics of Fibril Self-Assembly and Gelation Conformational Transition}

We begin by noting that the analysis below assumes that molecular weight of MC chains is sufficiently large to enable ring formation. We can estimate the threshold molecular weight as follows. If the size of a single glucose repeat unit (with molar mass approximately $188 \mathrm{~g} / \mathrm{mol}$ ) is $a \approx 0.5 \mathrm{~nm}$, and the outside radius of the ring $R \approx 7 \mathrm{~nm}$, the total number of repeat units in a single revolution is roughly $n \approx$ $2 \pi(R / a) \approx 90$, translating to molecular weight of about $16 \mathrm{~kg} / \mathrm{mol}$. In reality, the threshold should be probably higher (on the order of $25-30 \mathrm{~kg} / \mathrm{mol}$ ) since the walls could consist of more than one layer, according to the CG simulations. For polymers with molecular weight substantially larger than the threshold, the gelation temperature and the high-temperature modulus is nearly molecular-weight independent, as will be seen later. We now proceed to describe the theory.

The conformational transition from coils to rings, as seen in the simulation, strongly resembles the coil-helix transition, well studied in the past half-century, mainly in the context of polypeptides and proteins (though some carbohydrates also are known to possess helical structure in their crystalline state). ${ }^{27-34,36,54}$ The "inverted" coil-helix transition (coil at low temperatures, helix at elevated temperatures) has been observed in polypeptides such as poly-y-benzyl-L-glutamate in ethylene dichloride-dichloroacetic-acid mixtures. ${ }^{55}$ The theory of such transition is based on two premises. First, 
each repeat unit can be in one of two states, coil (hydrogen bonded with the solvent) or helix (hydrogen bonded with another repeat unit). Second, the entropy of the helix state is lower than that of the coil state, and the internal energy of the helix state is higher than that of the coil state. This can be caused by a balance between the intrapolymer hydrogen bonding, polymer-solvent hydrogen bonding, and solventsolvent hydrogen bonding. As the polymer chains adopt a helix or ring conformation, the polymer-solvent hydrogen bonds are replaced with the intrapolymer hydrogen bonds. The entropy increase for the solvent molecules exceeds the entropy loss for the polymers, and therefore, the total entropy in the helix (high-temperature conformation) is higher than in the coil (low-temperature conformation).

Within a mean-field approximation, fraction of repeat units belonging to helices (or, in our case, rings), is given by, $^{27,29,30}$

$$
f_{\text {ring }}=\frac{\exp \left(-\frac{\Delta G}{k_{\mathrm{B}} T}\right)}{1+\exp \left(-\frac{\Delta G}{k_{\mathrm{B}} T}\right)}=\frac{\exp \left(\frac{\Delta S_{0}}{k_{\mathrm{B}}}\left[\frac{T_{0}}{T}-1\right]\right)}{1+\exp \left(\frac{\Delta S_{0}}{k_{\mathrm{B}}}\left[\frac{T_{0}}{T}-1\right]\right)}
$$

Here, $k_{\mathrm{B}}$ is the Boltzmann's constant; we also defined the transition temperature, $T_{0}=\Delta H_{0} / \Delta S_{0}$. The free energy difference is given by,

$$
\Delta G=G_{\text {ring }}-G_{\text {coil }}=\Delta H_{0}-T \Delta S_{0}
$$

Because of the semi-flexible nature of the polymer, each "turn" of the helix contains a large number of repeat units, $n$ $\sim 90$, as discussed above. One can then apply coarsegraining so that the new repeat unit would consist of $n$ glucose repeat units; the enthalpy and entropy of transition are effectively normalized per these new repeat units (i.e., per one turn of the helix). At higher temperatures, $\Delta G<0$ and the helix/ring conformation is preferable, while at lower temperatures, $\Delta G>0$ and random coil configuration is preferred. At the transition, $\Delta G=0$, and coils and rings coexist. Equation 2 assumes that conformational transition is not "cooperative," i.e., fraction of rings at each temperature is independent of the MC concentration. This assumption is probably reasonable at low $(<2$ wt \%) MC concentrations but could become less accurate as the concentration is increased. Further complexity comes from the fact that if chains are sufficiently long, there can be coexistence between coil and helix (or ring) regions within a single chain; thus, in principle, one needs to make a distinction between the fraction of repeat units belonging to rings and the fraction of chains that have a ring configuration. For simplicity, for now we assume that the two are the same.

\section{Fibril Self-Assembly and Gelation}

Let us now turn our attention to self-assembly of rings into tubes. In this, we adapt the approach originally proposed by Zilman et al. ${ }^{43-45}$ This approach was originally developed to describe a transition from spherical micelles to cylindrical micelles and micellar networks in surfactant solutions, and the physics of those systems and the one considered here seem to have many common features. We assume all the" ends" or "faces" (below, we will drop the quotation marks and use those terms interchangeably) of the rings are identical, and have energy (in units of $k_{\mathrm{B}} T$ ) $\varepsilon_{1}$ in water, and a number density of $\rho_{1}$. (Here and in the following, we will be using a lattice model similar to that of Flory ${ }^{56}$ and Huggins ${ }^{57}$ in which the volume of a lattice cell is equal to the volume of the single ring, and number densities are expressed per lattice unit and thus are dimensionless). Any two ends could coalesce, and we label the number density of such coalesced ends $\rho_{2}$, and their energy $\varepsilon_{2}$. Similarly, when three ends coalesce and form a network junction, the density and energy are labeled $\rho_{3}$ and $\varepsilon_{3}$. These arrangements are shown in Figure 1(c) with red corresponding to the body of the tubes. The circle color corresponds to the number of coalesced ends (yellow $=1$, green $=2$, and purple $=3$ ). The number densities $\rho_{1}, \rho_{2}$, and $\rho_{3}$ obey the following constraint for any given total number density of ends, $v$ :

$$
\rho_{1}+2 \rho_{2}+3 \rho_{3}=v=\frac{2 f_{\text {ring }} \varphi}{\alpha}
$$

Here, $\phi$ is the methylcellulose volume fraction in water, and $\alpha \approx 0.4$ is the volume fraction of polymer in the tube (we assume that it is the same as in experiments of Arvidson et al. $^{18-22}$ ). The free energy density of the ensemble of rings/tubes in water can be written as follows,

$$
\begin{gathered}
\frac{f}{k_{\mathrm{B}} T}=\left\{\rho_{1} \ln \left(\frac{\rho_{1}}{e}\right)+\rho_{2} \ln \left(\frac{2 \rho_{2}}{e}\right)+\rho_{3} \ln \left(\frac{3 \rho_{3}}{e}\right)\right\}+ \\
\varepsilon_{1} \rho_{1}+\varepsilon_{2} \rho_{2}+\varepsilon_{3} \rho_{3}+K\left(\rho_{1}+2 \rho_{2}+3 \rho_{3}-v\right)
\end{gathered}
$$

Here $T$ is absolute temperature and $e(2.71828 \ldots)$ is the base of natural logarithms. The first three terms of eq 5 describe translational entropy, the next three terms are enthalpic contributions, and the last term is simply a Lagrange multiplier that accounts for constraint (4). By minimizing eq 5 with respect to $\rho_{1}, \rho_{2}, \rho_{3}$, and $K$, we obtain,

$$
\begin{gathered}
\rho_{1}=\exp \left[-K-\varepsilon_{1}\right] \\
2 \rho_{2}=\exp \left[-2 K-\varepsilon_{2}\right] \\
3 \rho_{3}=\exp \left[-3 K-\varepsilon_{3}\right] \\
\rho_{1}+2 \rho_{2}+3 \rho_{3}=v=\frac{2 f_{\text {ring }} \varphi}{\alpha}
\end{gathered}
$$

The effective energies of three configurations, $\varepsilon_{1}, \varepsilon_{2}$, and $\varepsilon_{3}$ are, of course, themselves functions of temperature. We can imagine that at lower temperatures, favorable water-polymer hydrogen bonding would make $\varepsilon_{1}$ relatively small, while at higher temperatures, it should increase very strongly. Since one can always arbitrarily shift the energy scale, it is convenient to set $\varepsilon_{2}=0 .{ }^{43}$ As discussed by Zilman et al., ${ }^{43}$ one can assume that $\varepsilon_{1}$ and $\varepsilon_{3}$ are linear functions of temperature, with $\varepsilon_{1}$ constant or increasing and $\varepsilon_{3}$ decreasing as the temperature is increased. We thus parameterize, $\varepsilon_{i}=p_{i}$ $+q_{i}\left(T-T_{\text {ref }}\right)$, where $i=1,3$, and $T_{\text {ref }}=25^{\circ} \mathrm{C}$. 
Equations $6 \mathrm{a}$ to $6 \mathrm{~d}$ allow one to evaluate all relevant variables as function of $\phi$. One way to do this would be to express everything in terms of $\rho_{1}$ by solving for $K$ in eq 6 a and substituting it into all the other equations. We can thus evaluate $\rho_{1}, \rho_{2}, \rho_{3}$, and the free energy. As the polymer concentration is increased, $\rho_{2}$ grows faster than $\rho_{1}$, and $\rho_{3}$ faster than $\rho_{2}$. This results in formation of high aspect ratio hollow fibrils (the aspect ratio is proportional to $\rho_{2}$ ) and their subsequent crosslinking into elastic network (crosslink density equal to $\rho_{3}$ ). The gelation transition corresponds to the point where the number of junctions exceeds the number of ends, $\rho_{3}>\rho_{1}$. As discussed by Zilman et al., ${ }^{43,44}$ this condition means that for each fibril, on average, there is at least one branch point (the number of "crosslinks" is greater than the number of "chain ends"). This criterion can be rewritten in terms of $\varepsilon_{1}$, and $\varepsilon_{3}$, $\exp \left(\varepsilon_{3}-\varepsilon_{1}\right)=2 \rho_{2}$. Above the gel point, the newly formed elastic network, combined with the tight entanglement network, gives rise to a dramatic increase in elastic modulus at high temperatures.

\section{Shear Elasticity of the Fibrillar Network}

McAllister et al. ${ }^{21,22}$ recently analyzed their experimental measurements of linear viscoelasticity of MC gels using the theory of entangled fibrillar networks. Within this approach-originally proposed by MacKintosh et al., ${ }^{47}$ the elastic shear modulus is given by,

$$
G=6 \rho k T l_{\mathrm{p}}^{2} \xi^{-3} \cong 6 \rho k T l_{\mathrm{p}}^{2}\left[\xi_{\mathrm{e}}^{-3}+\xi_{\mathrm{c}}^{-3}\right]
$$

Here, $l_{\mathrm{p}}$ is persistence length of the fibrils (not to be confused with persistence length of the MC chains themselves), $\rho$ is the areal density of the fibrils, and $\xi$ is effective "mesh size" or distance between crosslinks or entanglements. As discussed by MacKintosh et al., ${ }^{47}$ in the limit of high density of entanglements, $\xi_{\mathrm{e}} \cong R \psi^{-1 / 2}$, where $R$ is the fibril radius (assumed to be equal to the radius of the single-chain ring, as discussed above), and the effective fibril volume fraction is $\psi=\frac{f_{\text {ring }} \varphi}{\alpha}$. The crosslinks formed by the Y-junctions contribute another term, $\xi_{\mathrm{c}}^{-3} \cong R^{-3} \rho_{3}$, where $\rho_{3}$ is the density of Y-junctions. We note that in the limit of high entanglement density discussed here, both crosslinks and entanglements give rise to the same power-law behavior, $G \sim \phi^{2.5}$. Thus, to distinguish the two contributions unambiguously, one needs to analyze the rheology in more detail; this analysis is beyond the scope of the current paper and will be the subject of future studies. The areal density of fibrils, $\rho$, is given by, ${ }^{22}$

$$
\rho=\frac{f_{\text {ring }} \varphi}{\alpha \pi R^{2}}
$$

Combining all the pieces together, we can write down an expression for the shear modulus $G$,

$$
\begin{aligned}
G & =6 \frac{f_{\text {ring }} \varphi l_{\mathrm{p}}^{2}}{\alpha \pi R^{5}} k_{\mathrm{B}} T\left[\rho_{3}+\left(\frac{f_{\text {ring }} \varphi}{\alpha}\right)^{3 / 2}\right] \\
& \approx 6 \frac{f_{\text {ring }} \varphi l_{\mathrm{p}}^{2}}{\alpha \pi R^{5}} k_{\mathrm{B}} T\left(\frac{f_{\text {ring }} \varphi}{\alpha}\right)^{3 / 2}\left[\exp \left(-\varepsilon_{3}\right)+1\right]
\end{aligned}
$$

The first term in the square brackets in the right-hand side describes contributions from the permanent crosslinks and the second term contains those of entanglements. As the temperature is increased, the importance of the first term grows. At the same time, as discussed above, precise quantification of each term is impossible without more detailed analysis of frequency-dependent or time-dependent rheology. It is also important to emphasize that implicit in this model is the assumption that both the fibril density and the average fibril length are large enough to ensure the formation of a uniform, percolated network; thus, eq 10 can be applied to describe modulus only in the gel region (above gelation temperature for a particular MC concentration).

This concludes the description of the models. We now proceed to compare model predictions with experimental results.

\section{RESULTS AND DISCUSSION}

\section{Concentration-Dependent Assembly of Multiple Methylcellulose Chains into Rings}

Figure 3 depicts simulation starting from a random configuration of three $\mathrm{MC}$ chains with degree of polymerization $\mathrm{DP}=1000$ (molecular weight of $188 \mathrm{~kg} / \mathrm{mol}$, substitution type distribution given in Supporting Information, Table S3) at different concentrations. The cubic simulation box size was set to $400,180,80,60$, and $45 \mathrm{~nm}$ on the side, corresponding to concentrations of $0.002,0.017,0.19,0.46$, and 1.09 wt \%. The model polymer chains were randomly placed in the box [Fig. 3(a)] and allowed time for adequate equilibration at $25{ }^{\circ} \mathrm{C}$. The temperature was then increased to 50 ${ }^{\circ} \mathrm{C}$ and the final snapshots from each simulation at different concentrations are shown in Figure 3(b-f). At a very dilute concentration, namely 0.002 wt $\%$, individual chains formed isolated rings. These rings have been observed in a singlechain study, ${ }^{39,40}$ and the diameters of these rings are independent of the specific repeat unit sequence in the randomly substituted chains; the outer diameter is estimated to be $13.9 \pm 0.4 \mathrm{~nm}$ and the inner diameter is $7.1 \pm 0.2 \mathrm{~nm}$. The outer diameter compares well to that $(14 \pm 2 \mathrm{~nm})$ measured experimentally for long MC fibrils. Similarly, the inside diameter compares reasonably well to that $(10.8 \pm 3 \mathrm{~nm})$ estimated with the measured fibril diameter and water content assuming an ideal cylindrical hollow tube. Formation of ring structures has also been reported in a recent systematic coarse-graining study of isolated semiflexible polymer chain with strong self-interaction. ${ }^{40}$ Note that at a concentration of 0.002 wt $\%$, the isolated ring structures did not interact with each other during the course of simulation.

In a dilute solution, namely between 0.017 and 0.3 wt \%, at least one chain first formed an isolated single-chain ring structure, which we refer to as a "seed ring structure." The other chains, whether still in the random coil state or having also formed ring structures, then came into contact with the seed ring and fused with it, forming a single tubular structure. The "height" (extent in the direction parallel to the main axis) of the individual ring is $2.6 \pm 0.3 \mathrm{~nm}$. The single stable "proto-tube" structure formed by the three rings has 


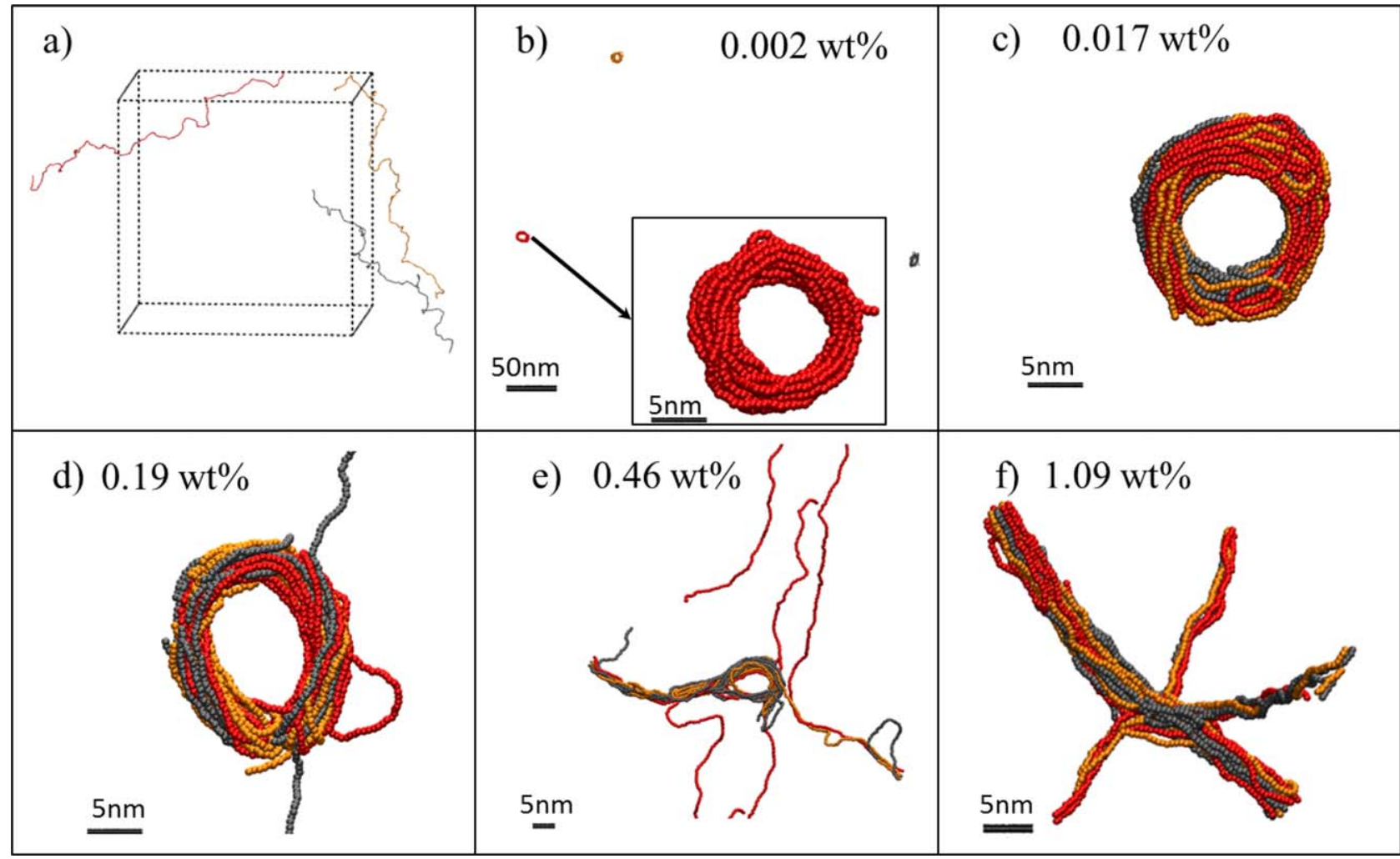

FIGURE 3 Snapshots from five simulations of chains with DP $=1000$ with varying polymer concentrations. (a) At the beginning, three chains are randomly placed in a cubic simulation box with periodic boundary conditions. To vary the concentration, we change the box size $L=400,180,80,60$, and $45 \mathrm{~nm}$, corresponding to polymer concentrations of $0.002,0.017,0.19,0.46$, and 1.09 wt \%. (b-f) Final snapshots of the simulations corresponding to various MC concentrations. All simulations were performed at $T=50^{\circ} \mathrm{C}$. [Color figure can be viewed in the online issue, which is available at wileyonlinelibrary.com.]

an outer diameter of $16.4 \pm 1.3 \mathrm{~nm}$, an inner diameter of $7.9 \pm 1.1 \mathrm{~nm}$, and a total height of $4.7 \pm 0.3 \mathrm{~nm}$. The ring is packed with many revolutions of methylcellulose CG beads. The thickness of the proto-tube wall increases by $1.25 \mathrm{~nm}$ on average after individual rings fuse, corresponding to two revolutions of CG beads, since each CG bead has a diameter of $0.515 \mathrm{~nm}$. The height of the three-molecule proto-tube is $2.1 \mathrm{~nm}$ more than the height of a single ring, corresponding to an addition of five revolutions in axial direction.

As the concentration of the polymer in solution increases to roughly between 0.5 and 1.1 wt $\%$, initially dispersed chains formed a bundle structure and evolved into a three dimensional network of bundles. Note that at $0.5 \mathrm{wt} \%$, bundles formed and bent into imperfect ring [Fig. 3(e)], suggesting another possible pathway to a ring structure. At 1.1 wt \% however, the bundles formed a three dimensional network of bundles with no rings. This three dimensional network is similar to the conventional gelation network for methylcellulose proposed by Kato et al., ${ }^{3}$ though not necessarily easy to reconcile with the fibrillar network observed experimentally. It is also possible, as hypothesized by McAllister et al., ${ }^{21}$ that both fibrils and bundles could be metastable structures, but formation of fibrils is preferred for kinetic reasons, for example by rapid growth from seed ring structures that are pres- ent in the solution due to compositional inhomogeneity. At this time, we do not have a clear explanation for why MC solutions of concentration greater than $1.1 \mathrm{wt} \%$ would form hollow fibers rather than fibrillar gels with fibers composed of bundles of parallel aligned MC molecules. If the latter were to occur, however, the fibril diameters would have no strongly preferred value and a distinct fibril diameter of $14 \mathrm{~nm}$ would not be expected, nor would the rather low (40\%) density of polymer in the fibers be explicable. Hence, for now we hypothesize that some thermodynamic or kinetic factor favors formation of rings even at concentrations above that for which our simulations predict them to form. We proceed, then, to simulate the assembly of such rings into tubular fibrils, and, following that, develop a thermodynamic theory for this assembly.

\section{Self-Assembly of Multiple Rings into a Proto-Tube}

Next, we examined how the rings could aggregate into a tubular structure. An initial ring structure was generated by simulating a 1000 -repeat unit chain at elevated $\left(50^{\circ} \mathrm{C}\right)$ temperature, and then replicated five times. We then performed several simulations in which the initial positions of the five rings were varied. In the first simulation [Fig. 4(a)], the replicates were initially placed on top of each other with $2 \mathrm{~nm}$ center-to-center distances and eventually fused into a single 


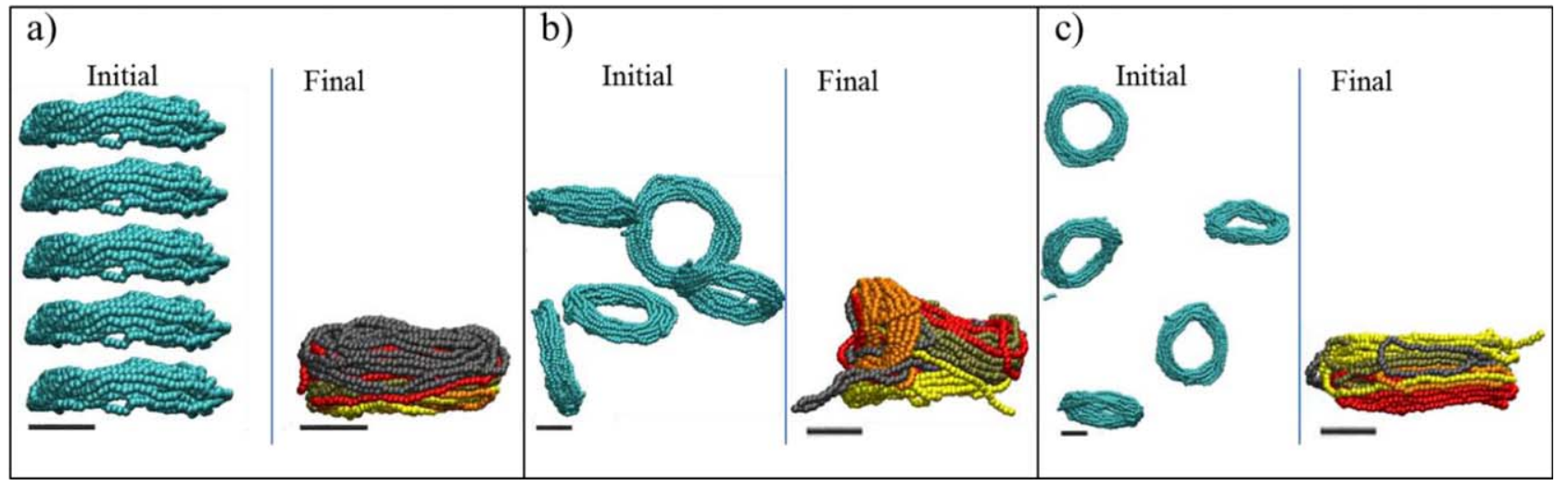

FIGURE 4 Initial and final snapshots from simulations of five replicated rings self-assembling into tubular structure. The ring is formed from a single heterogeneous $\mathrm{MC}$ chain with $\mathrm{DP}=1000$. All scale bars are $5 \mathrm{~nm}$. Three initial configurations were constructed. (a) Replicates are placed on top of each other. (b, c) Replicates are placed randomly in the simulation box at concentration of 3.9 and $1.3 \mathrm{wt} \%$ respectively. [Color figure can be viewed in the online issue, which is available at wileyonlinelibrary.com.]

proto-tube structure. In the second simulation [Fig. 4(b)], the rings were initially placed randomly and ended up forming a single ring, however with some defects. Those studies corresponded to the polymer volume fraction of $3.9 \mathrm{wt} \%$. By increasing the simulation box size, we reduced the effective concentration to $1.3 \mathrm{wt} \%$ [Fig. 4(c)], and observed that the rings self-assembled and fused into a single tube. This suggests that in heated methylcellulose solution, at lower polymer concentrations, single-chain rings self-assemble into tubular structures. At higher concentrations, the ring selfassembly is more likely to include branch points and other defects.

The proto-tubes, of course, can "polymerize" further, given the right conditions. For example, taking two five-ring structures and putting them one on top of another leads to the formation of a 10-chain tube [Fig. 5(a)]. The tube has an outer diameter of $17.4 \pm 0.6 \mathrm{~nm}$ and an inner diameter of $6.3 \pm 0.7 \mathrm{~nm}$ [Fig. 5(b)]. We calculate the void fraction in the center of this structure as the square of the ratio of inner diameter over the outer diameter. The void fraction is thus estimated to be approximately $13 \%$, which is substantially smaller than the water volume fraction observed in experiments. ${ }^{18-20}$ However, we note the effective density of coarsegrained repeat units is much lower than that of the polymer itself because of the coarse-graining which replaces flat repeat unit by spherical beads. We estimate each cellulosic repeat unit to be a thin cylindrical disk shape that has a height of $0.15 \mathrm{~nm}$ and diameter of $0.515 \mathrm{~nm}$. With this assumption, polymeric material occupies about $45 \%$ of volume, and the remaining 55\% are effectively interatomic voids. Assuming the wall of the ring is densely packed with CG beads, we can re-map the CG beads to atomistic MC repeat units, and thereby find that $52 \%$ of the total tubular structure volume will be voids and can be accessed by water molecule. Note however, that to simulate such a backmapped atomistic system with 5000 repeat units and explicit water molecules present is not currently possible. Therefore, while we do not know how the atomistic chains would pack if simulated de novo, this mapping calculation yields a

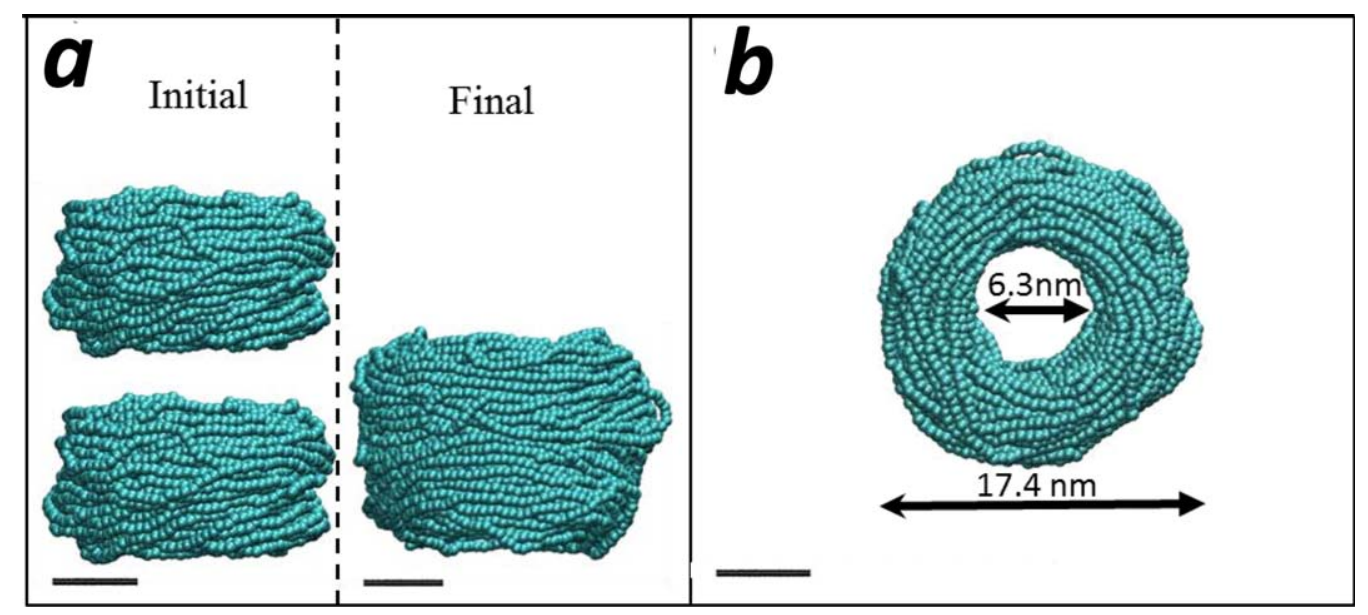

FIGURE 5 (a) Two five-chain proto-tubes are brought together and equilibrated, forming a 10-chain tube (b) Cross-section and dimensions of a three-chain proto-tube. All scale bars in the figure are $5 \mathrm{~nm}$. [Color figure can be viewed in the online issue, which is available at wileyonlinelibrary.com.] 


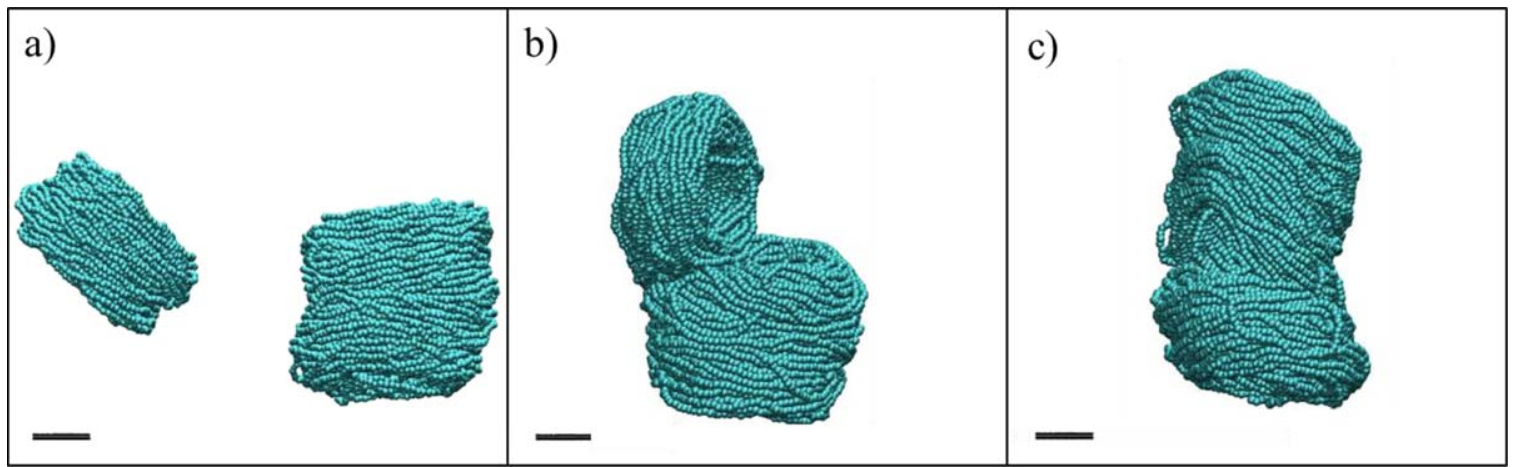

FIGURE 6 Snapshots of (a) initial, (b) metastable, and (c) final structures in the fifteen-chain tube structure growth simulations. All scale bars in the figure are $5 \mathrm{~nm}$. [Color figure can be viewed in the online issue, which is available at wileyonlinelibrary.com.]

reasonably good agreement with the $60 \%$ water content percentage reported in the experiments.

To further test if the proto-tube structures can self-assemble without a prearranged or ordered initial structure (e.g. the stacking configuration with a small separation), we set up a simulation with one five-chain proto-tube structure space $5 \mathrm{~nm}$ to the side of a ten-chain proto-tube structure [Fig. 6(a)]. The five-chain structure came into contact with the "cap" of the ten-chain structure and formed a metastable "proto-junction" [Fig. 6(b)]. This metastable structure persisted for around 100 ns before rearranging to form a single, higher aspect-ratio tube [Fig. 6(c)]. We hypothesize that if another proto-tube were nearby when the metastable structure is formed, the three proto-tubes could form a stable three-way junction, as illustrated in Figure 1(c).

To quantify the dimensions of the tubular structures more accurately, we used the radius of gyration tensor approach. The eigenvalues of the radius of gyration tensor are expressed in the following equation:

$$
R_{\mathrm{g}}^{2}=\lambda_{x}^{2}+\lambda_{y}^{2}+\lambda_{z}^{2}
$$

The eigenvalues are ordered such that $\lambda_{x}^{2} \geq \lambda_{y}^{2} \geq \lambda_{z}^{2}$. We plot in Figure 7 the averaged eigenvalues for all final ring and tube structures obtained from simulations. Two eigenvalues, namely $\lambda_{x}$ and $\lambda_{y}$, have very similar values in all structures. These two values correspond to the outer diameter of the axisymmetric ring and tube. Note that these two values increase upon transitioning from a one-chain to a five-chain proto-tubular structure, confirming the growth of the tubular structure's outer diameter observed through visual inspection. Among 5-, 10-, and 15-chain tubular structures, these two eigenvalues are very consistent, indicating the tube structure's diameter does not change any further. More importantly, the $\lambda_{z}$ value, which corresponds to the height of the ring and tube structures, increases almost linearly from a 1-chain to a 15-chain tube, clearly indicating the tubular structure grows axially once its stable inner and outer diameters are established. Our simulation results thus support the proposed gelation mechanism in the theoretical model outlined below. Individual chains in a random coil state undergo a conformational transition when temperature rises and form isolated ring structures. These ring structures, similar to nucleation sites in crystal growth model, attract other rings and self-assemble into short proto-tubes, and eventually grow into long hollow (water-filled) fibrils with a uniform inner and outer diameter. In our multiple chains simulations, the outer diameter of all tubular structures ranges from 14 to $17 \mathrm{~nm}$, in excellent agreement with $15 \pm$ $2 \mathrm{~nm}$ as reported by Arvidson et al. ${ }^{18-21}$

\section{Stability of the Tubular Structure under Cooling/Heating Cycle}

Finally, we demonstrate that the formation of the tubular structure at elevated temperature can be reversed by cooling the ten-chain tubular structure obtained from simulations described in the previous section [Fig. 8(a)]. To do so, we lowered the system temperature to an arbitrary medium temperature, which is slightly below the gelation temperature. This is achieved by lower the $\varepsilon_{i i}$ values derived at $50^{\circ}$ C by $20 \%$, assuming $\varepsilon_{i i}$ values scale linearly with the temperature (Supporting Information, Table S2). In response, the

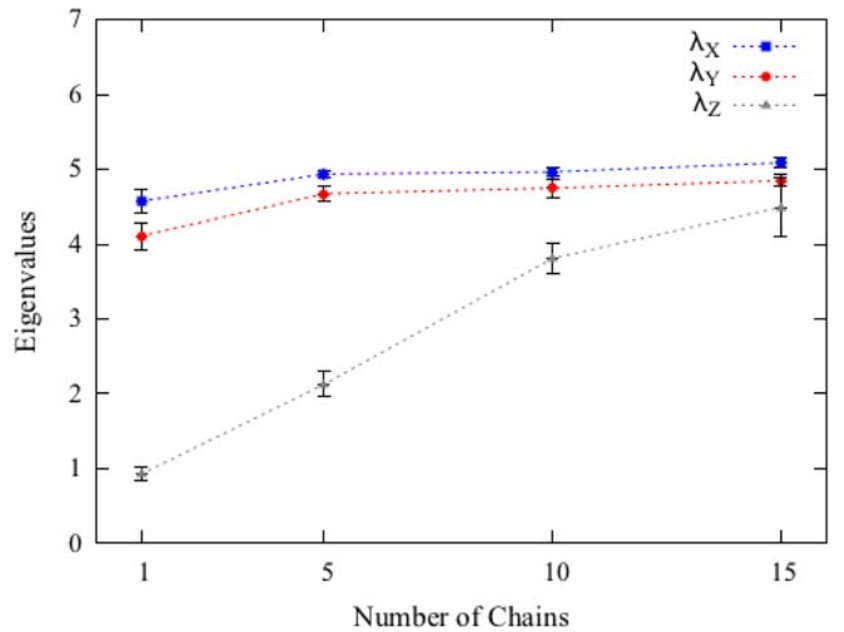

FIGURE 7 Three averaged eigenvalues $\left(\lambda_{x}, \lambda_{y}, \lambda_{z}\right.$ in $\left.\mathrm{nm}\right)$ of the radius of gyration tensor as a function of number of chains in the ring and tube structures. [Color figure can be viewed in the online issue, which is available at wileyonlinelibrary.com.] 


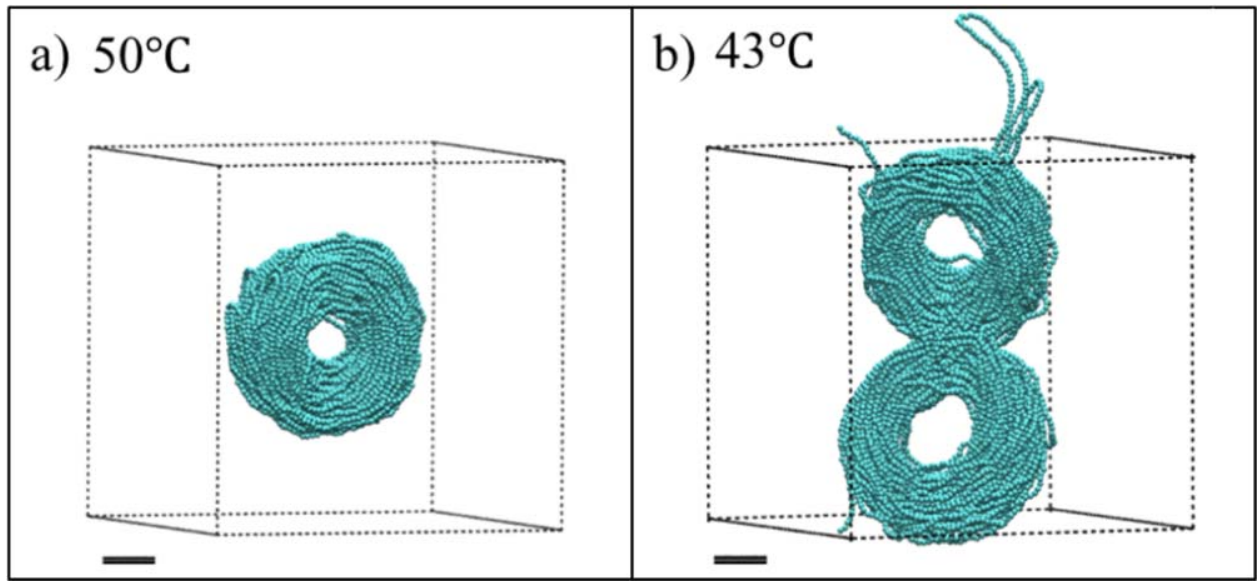

FIGURE 8 Snapshots of 10 -chain tube structure at a) $50{ }^{\circ} \mathrm{C}$ and b) $43{ }^{\circ} \mathrm{C}$. All scale bars in the figure are $5 \mathrm{~nm}$. [Color figure can be viewed in the online issue, which is available at wileyonlinelibrary.com.]

single ten-chain tubular structure broke into two smaller tubular structures with similar outer and inner diameters. Due to the effect of periodic boundary condition, the two proto-tubes remained in contact with the periodic images and therefore cannot move further apart [Fig. 8(b)]. This suggests that when temperature is lowered below the gelation temperature, segments of tubular structures will remain in solution and recombine when temperature is raised to above the gelation temperature again. Indeed, we recovered the original single ten-ring tube structure when we increased the system temperature back to $50{ }^{\circ} \mathrm{C}$, mimicking the cooling and heating cycle in a typical MC gelation experiment.

\section{Calculation of Gel Point and Rheology and Comparison with Experiments}

To compare our theory with experimental data, we begin by considering the conformational transition. As discussed by McAllister et al., ${ }^{21}$ the second virial coefficient $A_{2}$ of dilute MC solutions is observed to be positive at temperatures below approximately $48{ }^{\circ} \mathrm{C}$, and negative at higher temperatures. We can assume that the measured $A_{2}$ is the sum of positive contribution from coils and negative contribution

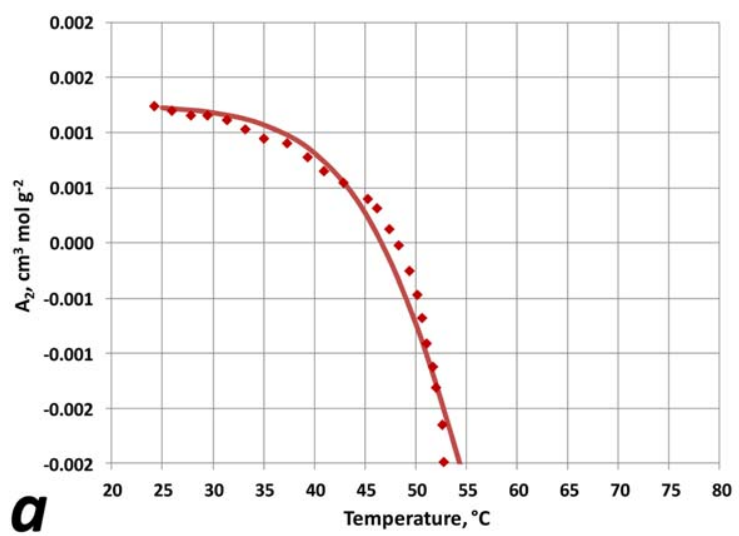

from the rings (not yet associated into fibrils). Then, from the dependence of $A_{2}$ on temperature, we can estimate the ring fraction,

$$
A_{2}=A_{2}^{\text {coil }}\left(1-f_{\text {ring }}\right)+A_{2}^{\text {ring }} f_{\text {ring }}
$$

Here, $f_{\text {ring }}$ is given by eq 3 . By fitting eqs 3 and 12 to the experimental dependence of $A_{2}$ on temperature [Fig. 9(a)], we can obtain model parameters related to the conformational transition (see Table 1) and estimate the fraction of rings as a function of temperature [Fig. 9(b)]. Most of the methylcellulose remains in the form of coils at temperatures below $\sim 55{ }^{\circ} \mathrm{C}$, although the fraction of rings is no longer negligible at temperatures above $\sim 45{ }^{\circ} \mathrm{C}$. The latter means that at moderate-to-high polymer concentrations, there is enough material to form fibrils provided that the solution is aged long enough for fibril nucleation and growth.

Next, we compute the gelation temperature as function of MC concentration. We have assumed that $\varepsilon_{1}$ and $\varepsilon_{3}$ depend on temperature in a linear fashion and chose the energy scale in which $\varepsilon_{2}=0$ at all temperatures. By fitting the

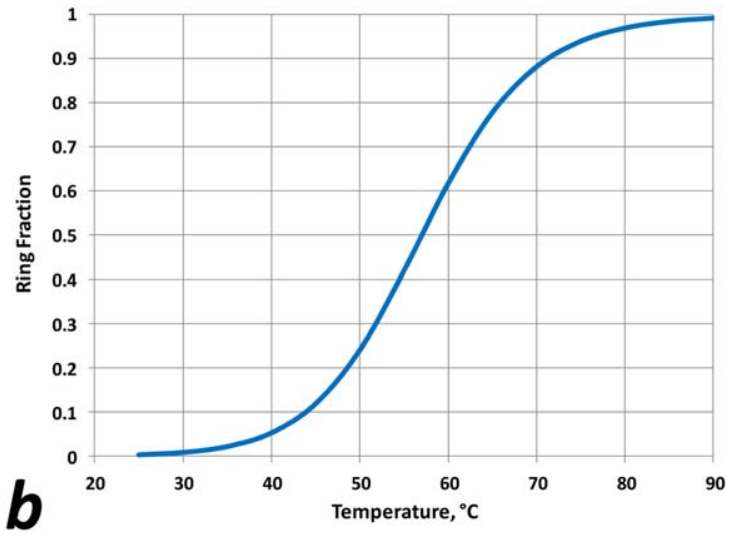

FIGURE 9 (a) Second virial coefficient, $A_{2}$, as function of temperature. Symbols-experimental data of Lott et al.; ${ }^{20}$ line is the best fit according to eq 9. (b) Predicted ring fraction as function of temperature (eq 2). See text for more detail. [Color figure can be viewed in the online issue, which is available at wileyonlinelibrary.com.] 
TABLE 1 Parameters Related to Gelation and Rheology Models

\begin{tabular}{ll}
\hline Parameter (Units) & Value \\
$T_{0}\left({ }^{\circ} \mathrm{C}\right)$ & 57.0 \\
$\Delta S_{0} / k_{\mathrm{B}}$ & 53.0 \\
$A_{2, \text { coil }}\left(\mathrm{cm}^{3} \mathrm{~mol} \mathrm{~g}^{-2}\right)$ & $1.25 \times 10^{-3}$ \\
$A_{2, \text { ring }}\left(\mathrm{cm}^{3} \mathrm{~mol} \mathrm{~g}^{-2}\right)$ & $-7.0 \times 10^{-3}$ \\
$p_{1}$ & 7.0 \\
$q_{1}\left(\mathrm{~K}^{-1}\right)$ & 0 \\
$p_{3}$ & 59.15 \\
$q_{3}\left(\mathrm{~K}^{-1}\right)$ & -0.175 \\
$l_{\mathrm{p}}(\mathrm{nm})$ & 48.0 \\
$\alpha$ & 0.4
\end{tabular}

lower-concentration portion of experimental data of Arvidson et al. ${ }^{18}$ (Fig. 10), we can estimate the energy difference $\varepsilon_{3}-\varepsilon_{1}$ as function of temperature (see Fig. 11). As expected, the model works well at low MC concentrations, showing a linear decrease of the gelation temperature with $\log \left(\phi_{\mathrm{MC}}\right)$. At higher concentrations, the model underestimates the gel temperature-once again, this could be due to cooperativity between the polymer chains influencing the onset of the conformational transition. This result is qualitatively similar to what was found for surfactant micellar solutions. ${ }^{43}$

Finally, we consider the shear rheology $\left(G^{\prime}\right)$ as function of temperature. To do this, we apply eq 10 to experimental measurements of the shear storage modulus at different temperatures and concentrations. From gelation temperature estimates discussed above, we determined the difference $\varepsilon_{3}$ $-\varepsilon_{1}$ as function of temperature; by fitting the $G^{\prime}$ data for multiple temperatures and concentrations, we obtain $\varepsilon_{3}$ (as function of temperature) and the fibril persistence length, $l_{\mathrm{p}}$.

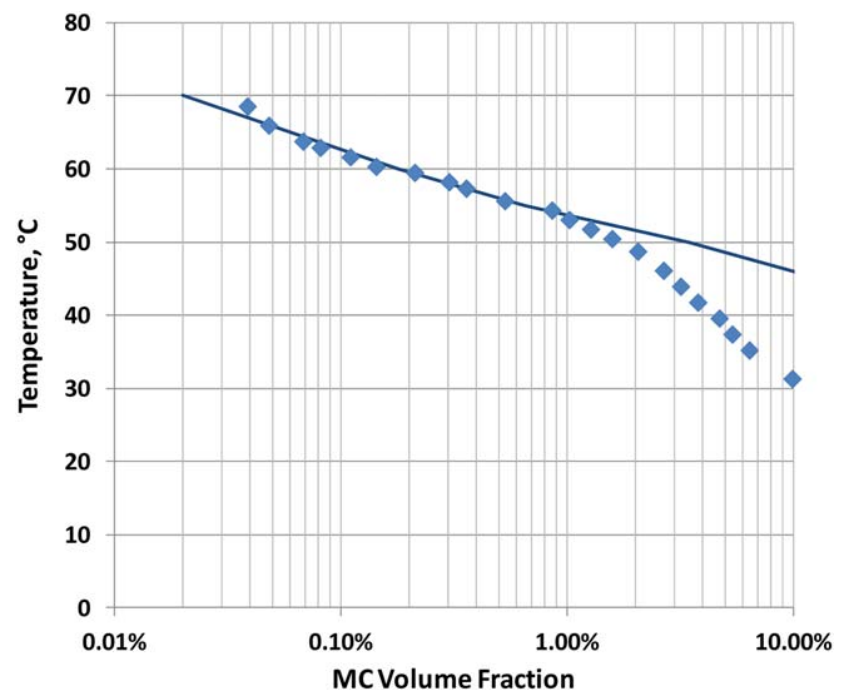

FIGURE 10 Gelation temperature as function of $\mathrm{MC}$ volume fraction. Symbols are the data from Arvidson et al. ${ }^{18}$ and the line is the model fit. [Color figure can be viewed in the online issue, which is available at wileyonlinelibrary.com.]

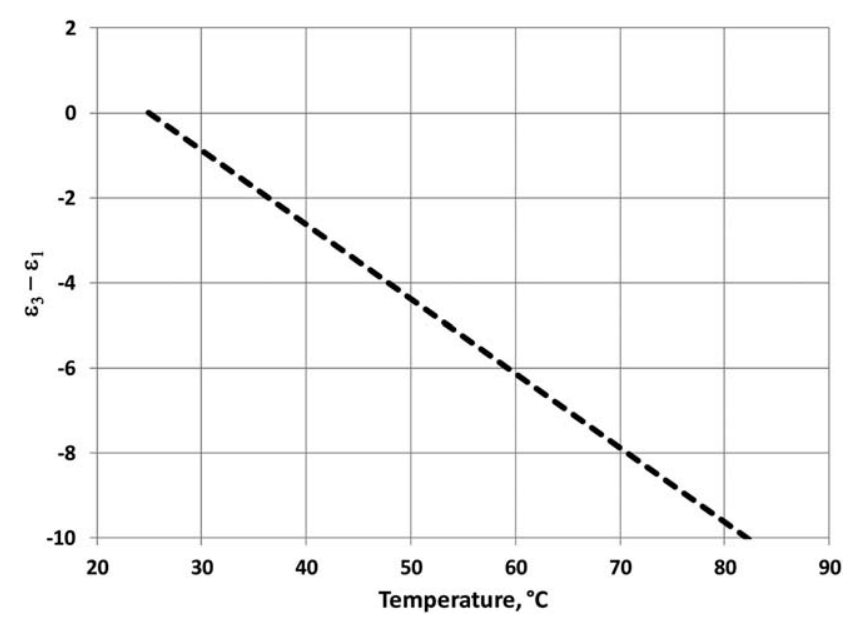

FIGURE 11 Effective energy difference (in units of $k_{\mathrm{B}} T$ ) between $\mathrm{Y}$-junctions and ends, as function of temperature.

The fibril end energy $\varepsilon_{1}$ can then be calculated as $\varepsilon_{1}(T)=\varepsilon_{3}$ $(T)-\left(\varepsilon_{3}(T)-\varepsilon_{1}(T)\right)$ where the first term in the right-hand side is estimated from rheology fitting and the second term from the gelation fitting. Based on this analysis, we can see that the fibril end energy is positive and approximately temperature-independent, $\varepsilon_{1} \approx 7.0$ (in units of $k_{\mathrm{B}} T$ ). Figure 12(a) shows the measured $G^{\prime}(T)$ heating curves (based on results of Arvidson et al. ${ }^{18}$ ) and corresponding modeling fits for three MC concentrations $(0.7 \%, 1.4 \%$, and $2.8 \%)$ for one MC material $\left(M_{\mathrm{w}}=300 \mathrm{~kg} / \mathrm{mol}, \mathrm{DS}=1.8 \mathrm{~mol} \mathrm{MeO} / \mathrm{mol}\right.$ AGU). The high-temperature rheology is correctly captured by the model, as one can see in Figure 12(b) where the data from Arvidson et al. ${ }^{18}$ for $G^{\prime}$ as function of MC concentration at $T=80{ }^{\circ} \mathrm{C}$ are plotted against the model calculation. The model predicts high-temperature scaling of $G \sim(\phi)^{2.5}$ as discussed above, and this scaling is in very good agreement with experimental data, as indeed was already discussed by McAllister et al. $^{22}$ Our analysis suggests that the elasticity of fibrillar gels is due to both entanglements and permanent crosslinks (Y-junctions), with the contribution of permanent crosslinks increasing and dominating at high temperatures. Model parameters are summarized in Table 1 (as a reminder, we assumed linear dependence of the end and crosslink energies on temperature, $\varepsilon_{i}=p_{i}+q_{i}\left(T-T_{\text {ref }}\right)$, where $i=1$, 3 , and $T_{\text {ref }}=25^{\circ} \mathrm{C}$ ).

The above analysis does not necessarily prove that the aqueous MC fibrils are indeed hollow self-assembled tubes; other hypotheses are also possible. In fact, our CG simulations suggest that networks of fibers containing varying numbers of parallel MC chains would be expected to form at concentrations where polymer chains strongly overlap. It is thus possible that, as proposed by McAllister et al., ${ }^{21}$ the rings and hollow tubules are metastable structures favored for kinetic reasons, while bundles and liquid-crystalline fibers are equilibrium but kinetically hindered morphologies. What is shown, however, is that if the ring-like structures do indeed form at high as well as low MC concentration, then their assembly into networks of hollow tubules is consistent with 

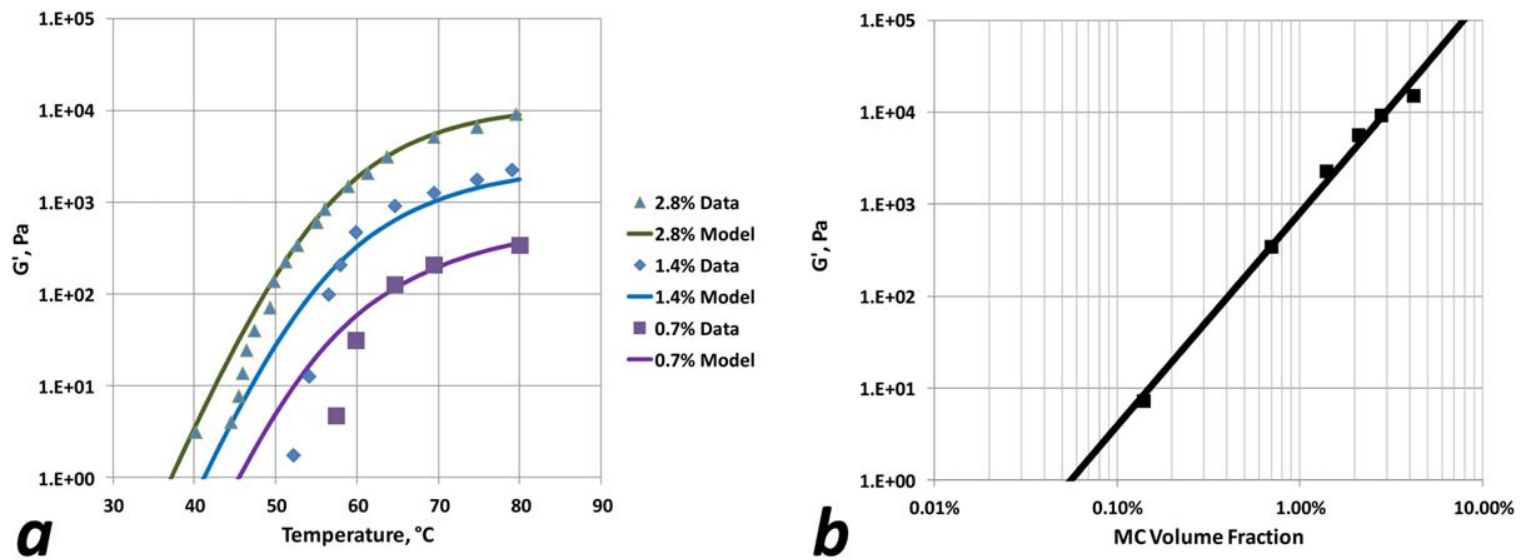

FIGURE 12 (a) Shear storage modulus $G^{\prime}$ measured as function of temperature for several MC concentrations (polymer $M_{\mathrm{w}}=300 \mathrm{~kg} / \mathrm{mol}$, DS = 1.8). The data are from Arvidson et al., ${ }^{18}$ and lines represent model fits to the data. (b) Shear storage modulus $G^{\prime}$ as function of methylcellulose volume fraction. The data are measured at $80{ }^{\circ} \mathrm{C}$ (Arvidson et al. ${ }^{18}$ ). The line is the model fit to the data (see text for more details). [Color figure can be viewed in the online issue, which is available at wileyonlinelibrary. com.]

both experimental data and our CG simulations, and provides qualitative and semi-quantitative description of thermodynamics and rheology of aqueous MC systems.

\section{CONCLUSIONS}

In summary, we proposed a new multi-scale model of aqueous methylcellulose gelation. Using coarse-grained molecular dynamics (CG-MD), we showed that individual MC chains in water undergo conformational change from coil to ring upon increase in temperature. We further observed that the rings subsequently self-assemble into stacks or "proto-fibrils" and that sometimes, can give rise to Y-junctions. We then developed a statistical-mechanical model to describe the gelation transition and the dependence of elastic modulus on temperature and concentration. The model successfully explains several important experimental observations. First, sol-gel transition temperature is more or less independent of polymer molecular weight once it exceeds certain threshold. Second, gel modulus at high temperature grows with $\mathrm{MC}$ volume fraction as a power-law function with exponent close to 2.3 (our theory predicts the exponent of 2.5). Third, gelation temperature increases as the MC concentration is reduced. Fourth, the fibril outside and inside diameters are set by the ring conformation. Fifth, fibrils can be observed (after aging of several hours or days) in systems where polymer and water are supposed to be miscible based on the sign of the second virial coefficient $A_{2}$ (i.e., at temperatures between 40 and $48{ }^{\circ} \mathrm{C}$ ). The model is also able to describe gel point transition and modulus dependence on temperature and concentration in a semiquantitative fashion. Yet, many questions also remain. What are the molecular mechanisms that drive the conformational transition? Why do ringlike structures form at high polymer concentrations where the molecules can instead form parallel bundles and thereby avoid the chain bending energy that must be paid to form a ring? How can we predict the effective interaction energies of rings and coils with water as a function of temperature? Can we predict the dynamics of fibril self-assembly, again as function of temperature, concentration, and heating rate? What about hysteresis between heating and cooling? These-and many other topics-will be the subject of future work, and will provide further insight into this interesting scenario of anisotropic polymer self-assembly.

\section{ACKNOWLEDGMENTS}

We thank Tim Lodge, Frank Bates, Sarah Arvidson, Joe Lott, John McAllister (University of Minnesota), Robert Schmitt, William Porter III, Tirtha Chatterjee, and Jonathan Moore (Dow) for many helpful and stimulating discussions. This work was supported by The Dow Chemical Company through grant 223278AF. Computational resources and services were provided in part by the Advanced Research Computing at the University of Michigan, Ann Arbor, and in part through the Extreme Science and Engineering Discovery Environment (XSEDE, grant number TG-CHE140009), which is supported by National Science Foundation grant number ACI-1053575.

\section{REFERENCES AND NOTES}

$1 \mathrm{~S}$. Kamel, N. Ali, K. Jahangir, S. M. Shah, A. A. El-Gendy, Express Polym. Lett. 2008, 2, 758-778.

2 P. Varela, S. M. Fiszman, Food Hydrocoll. 2011, 25, 18011812.

3 T. Kato, M. Yokoyama, A. Takahashi, Colloid Polym. Sci. 1978, 256, 15-21.

4 N. Sarkar, J. Appl. Polym. Sci. 1979, 24, 1073-1087.

5 A. Haque, E. R. Morris, Carbohydr. Polym. 1993, 22, 161-173.

6 C. Chevillard, M. A V. Axelos, Colloid Polym. Sci. 1997, 275, 537-545.

7 J. Desbrieres, M. Hirrien, S. B. Ross-Murphy, Polymer 2000, $41,2451-2461$ 
8 C. Clasen, W. M. Kulicke, Prog. Polym. Sci. 2001, 26, 18391919.

9 C. M. Keary, Carbohydr. Polym. 2001, 45, 293-303.

10 L. Li, P. M. Thangamathesvaran, C. Y. Yue, K. C. Tam, X. $\mathrm{Hu}, \mathrm{Y}$. C. Lam, Langmuir 2001, 17, 8062-8068.

11 M. Takahashi, M. Shimazaki, J. Yamamoto, J. Polym. Sci. Part B: Polym. Phys. 2001, 39, 91-100.

12 L. Li, H. Shan, C. Y. Yue, Y. C. Lam, K. C. Tam, X. Hu, Langmuir 2002, 18, 7291-7298.

13 T. Chatterjee, A. I. Nakatani, R. Adden, M. Brackhagen, D. Redwine, H. W. Shen, Y. F. Li, T. Wilson, R. L. Sammler, Biomacromolecules 2012, 13, 3355-3369.

14 J. P. A. Fairclough, H. Yu, O. Kelly, A. J. Ryan, R. L. Sammler, M. Radler, Langmuir 2012, 28, 10551-10557.

15 M. Knarr, R. Adden, W. H. K. Anderson, B. Hubner-Keese, Food Hydrocoll. 2012, 29, 317-325.

16 R. Bodvik, A. Dedinaite, L. Karlson, M. Bergstrom, P. Baverback, J. S. Pedersen, K. Edwards, G. Karlsson, I. Varga, P. M. Claesson, Colloids Surf. A 2010, 354, 162-171.

17 K. Kobayashi, C. I. Huang, T. P. Lodge, Macromolecules 1999, 32, 7070-7077.

18 S. A. Arvidson, J. R. Lott, J. W. McAllister, J. Zhang, F. S. Bates, T. P. Lodge, R. L. Sammler, Y. Li, M. Brackhagen, Macromolecules 2013, 46, 300-309.

19 J. R. Lott, J. W. McAllister, S. A. Arvidson, F. S. Bates, T. P. Lodge, Biomacromolecules 2013, 14, 2484-2488.

20 J. R. Lott, J. W. McAllister, M. Wasbrough, R. L. Sammler, F. S. Bates, T. P. Lodge, Macromolecules 2013, 46, 9760-9771.

21 J. W. McAllister, P. W. Schmidt, K. D. Dorfman, T. P. Lodge, F. S. Bates, Macromolecules 2015, 48, 7205-7215.

22 J. W. McAllister, J. R. Lott, P. W. Schmidt, R. L. Sammler, F. S. Bates, T. P. Lodge, ACS Macro Lett. 2015, 4, 538-542.

23 J. Yang, Curr. Opin. Colloid Interface Sci. 2002, 7, 276-281.

24 P. Akcora, H. Liu, S. K. Kumar, J. Moll, Y. Li, B. C. Benicewicz, L. S. Schadler, D. Acehan, A. Z. Panagiotopoulos, V. Pryamitsyn, Nat. Mater. 2009, 8, 354-359.

25 A. De Candia, E. Del Gado, A. Fierro, N. Sator, M. Tarzia, A. Coniglio, Phys. Rev. E 2006, 74, 010403.

26 X. Wang, D. S. Miller, E. Bukusoglu, J. J. De Pablo, N. L. Abbott, Nat Mater. 2015, 10, 1038. /nmat4421.

27 B. H. Zimm, J. K. Bragg, J. Chem. Phys. 1959, 31, 526-535.

28 S. Lifson, A. Roig, J. Chem. Phys. 1961, 34, 1963-1974.

29 M. Baur, L. Nosanow, J. Chem. Phys. 1962, 37, 153-160.

30 M. Baur, L. Nosanow, J. Chem. Phys. 1963, 38, 578-582.

31 S. Lifson, B. H. Zimm, Biopolymers 1963, 1, 15-23.

32 J. Gornall, E. Terentjev, Phys. Rev. Lett. 2007, 99, 028304.
33 J. L. Gornall, E. M. Terentjev, Soft Matter 2008, 4, 544-549.

34 A. Badasyan, S. A. Tonoyan, A. Giacometti, R. Podgornik, V. A. Parsegian, Y. S. Mamasakhlisov, V. F. Morozov, Phys. Rev. E 2014, 89, 022723.

35 C. R. Boehm, E. M. Terentjev, Macromolecules 2014, 47, 6086-6094.

36 A. Badasyan, Y. S. Mamasakhlisov, R. Podgornik, V. A. Parsegian, J. Chem. Phys. 2015, 143, 014102.

37 B. Schnurr, F. Mackintosh, D. Williams, Europhys. Lett. 2000, 51, 279.

38 B. Schnurr, F. Gittes, F. Mackintosh, Phys. Rev. E 2002, 65, 061904 .

39 M. O. Kong, I. S. Dalal, G. X. Li, R. G. Larson, Macromolecules 2014, 47, 1494-1502.

40 W. Huang, R. Ramesh, P. K. Jha, R. G. Larson, Macromolecules 2016, 49, 1490-1503.

41 Y. Bao, H. J. Qian, Z. Y. Lu, S. Cui, Macromolecules 2015, $48,3685-3690$.

42 S. Guillot, D. Lairez, M. Axelos, J. Appl. Crystallogr. 2000, 33, 669-672.

43 A. Zilman, S. Safran, T. Sottmann, R. Strey, Langmuir 2004, 20, 2199-2207.

44 A. Zilman, S. Safran, Phys. Rev. E 2002, 66, 051107

45 T. Tlusty, S. Safran, Science 2000, 290, 1328-1331.

46 T. Tlusty, S. Safran, J. Phys. 2000, 12, A253.

47 F. C. Mackintosh, J. Kas, P. A. Janmey, Phys. Rev. Lett. 1995, 75, 4425-4428.

48 F. Gittes, B. Schnurr, W. Moehler, P. Olmsted, J. Shah, M. Osterfield, P. Janmey, F. Mackintosh, C. Schmidt, Biophys. J. 1998, 74(2), A361-A361.

49 M. Gardel, J. Shin, F. Mackintosh, L. Mahadevan, P. Matsudaira, D. Weitz, Science 2004, 304, 1301-1305.

50 C. Joly-Duhamel, D. Hellio, A. Ajdari, M. Djabourov, Langmuir 2002, 18, 7158-7166.

51 S. Plimpton, J. Comput. Phys. 1995, 117, 1-19.

52 F. Müller-Plathe, ChemPhysChem 2002, 3, 754-759.

53 D. Reith, M. Pütz, F. Müller-Plathe, J. Comput. Chem. 2003, 24, 1624-1636.

54 L. Schefer, I. Usov, R. Mezzenga, Biomacromolecules 2015, 16, 985-991.

55 B. Zimm, P. Doty, K. Iso, Proc. Natl. Acad. Sci. USA 1959, 45, 1601.

56 P. J. Flory, J. Chem. Phys. 1941, 9, 660-660.

57 M. L. Huggins, J. Chem. Phys. 1941, 9, 440-440. 